\title{
Analysis of the beta-globin gene in DNA of suspected thalassemic great apes
}

\author{
J. Vuch ${ }^{1}$, M.S. Siori ${ }^{2}$, M.P. Bigatti ${ }^{2}$, L. Segat ${ }^{1}$, G. De Fabrizio ${ }^{4}$ and \\ S. Crovella ${ }^{3}$ \\ 'Institute for Maternal and Child Health, IRCCS "Burlo Garofolo", \\ Trieste, Italy \\ ${ }^{2}$ Department of Human and Animal Biology, University of Turin, Turin, Italy \\ ${ }^{3}$ Departamento de Genética, Universidade Federal de Pernambuco, \\ Recife, PE, Brasil \\ ${ }^{4}$ University of Trieste, Trieste, Italy \\ Corresponding autor: S. Crovella \\ E-mail: crovelser@gmail.com
}

Genet. Mol. Res. 12 (2): 1731-1739 (2013)

Received June 5, 2012

Accepted March 2, 2013

Published May 21, 2013

DOI http://dx.doi.org/10.4238/2013.May.21.4

\begin{abstract}
DNA was recovered from teeth of 2 great ape skeletons, Pan troglodytes (Ptr) and Pongo pygmaeus (Ppy), belonging to a 19thcentury zoological collection. The skeletons presented morphological alterations possibly associated with $\beta$-thalassemia: Ptr had deformation of the calvaria and oro-maxillo-facial bones with porotic hyperostosis and extended osteoporotic lesions of the skeleton, while Ppy showed a general marked widening of the calvarial diploe but moderate osteoporotic signs on the post-cranial skeleton. We screened Ptr and Ppy for mutations in the $\beta$-globin gene (exons 1,2 , and 3 ) because we suspected thalassemia. Ptr $\beta$-globin sequences showed the highest degree of similarity with the human ones (99.8\%), while those of Ppy were slightly different $(98.2 \%)$. The results were consistent with the phylogenetic relationships between their $\beta$-globin gene sequences. We did not find any mutation in the $\beta$-globin gene of Ptr and Ppy;
\end{abstract}


therefore, we conclude that, in spite of skeletal alterations, the 2 subjects analyzed were not affected by $\beta$-thalassemia.

Key words: Ancient DNA; $\beta$-globin gene; $\beta$-thalassemia; Mutation; Pan troglodytes; Pongo pygmaeus

\section{INTRODUCTION}

The analysis of ancient DNA sequences contributed in the last 20 years to successful clarification of phylogenetic and phylogeographic questions, providing a powerful tool for genetic population studies and allowing the analysis of the extinct species genome (Roy et al., 1994; Willerslev and Cooper, 2005; Rascol et al., 2007; Miller et al., 2008). The specimens conserved in museums represent an advantageous source for sampling of biological material from different species otherwise difficult to be recovered in nature or necessitating a lot of time-consuming fieldwork. Furthermore, when studying extinct species or analyzing populations that changed their structure over the time, museum specimens are unique biological sample sources (Roy et al., 1994).

Several methods for DNA extraction from bones and teeth have been published in the last few years (Rohland and Hofreiter, 2007a,b). Most of the methods are destructive of parts of bones or teeth; however, some non-destructive methods have been reported (Rohland et al., 2004; Rohland and Hofreiter, 2007b). Since the damage of museum specimens represents the "price" paid for getting biological material, most of the museums, in order to protect their collections, have restricted the permissions to use their specimens.

Another problem that characterizes archival/ancient DNA studies is the small amount of DNA that can be recovered due to preservation and degradation processes. Consequently, DNA availability and quality are the most important issues of ancient DNA studies. The most frequently used technology for ancient DNA analysis is polymerase chain reaction (PCR), even if alternative approaches such as ligation into bacterial vectors or addition of oligonucleotides for direct sequencing have been reported (Rohland and Hofreiter, 2007b). PCR amplification is a crucial issue since DNA extracted from archival materials such as bones and teeth may contain PCR inhibitors, which may interfere with the amplification, or "exogenous DNAs" co-purified with ancient DNA. In addition, the preservation of cell structures is very limited and DNA can be damaged or present chemical modifications. Thus, aggressive methods for DNA extraction should be avoided since they could diminish the overall DNA yield by causing additional damage to the DNA molecule.

In our study, we extracted DNA from the teeth of 2 great apes [Pan troglodytes (Ptr) and Pongo pygmaeus (Ppy)] obtained from museum collections where they have been preserved since 1800. We amplified and sequenced the coding region of the Ptr and Ppy $\beta$-globin gene with the aim of detecting mutations associated with $\beta$-thalassemia.

Both skeletons presented morphological alterations that lead us to hypothesize the presence of $\beta$-thalassemia: Ptr had widespread osteoporotic lesions of the skeleton with porotic hyperostosis, deformation of the calvaria and oro-maxillo-facial bones, and the "hair-on-end" phenomenon, while Ppy showed remarkable marrow hyperplasia of the skull and moderate osteoporotic signs on the post-cranial skeleton. Such osteopathologies have been well described in humans as suggestive of genetic anemias, likely thalassemia (Moseley, 1974; Stuart-Macadam, 1987; Hes et al., 1990; De Mattia et al., 1996; Hollar, 2001; Lagia et al., 2007), but to our knowledge, these 
cases have never been recorded in non-human primates, with the sole exceptions of Schultz (1956), Nathan and Haas (1966), and DeGusta (2010). While the allelic variants of the $\beta$-globin gene associated with thalassemia are very well known in different human ethnic groups (http://omim.org/ entry/141900), no mutations have been reported for the closely related great apes.

\section{MATERIAL AND METHODS}

The skeletons of Ptr and Ppy belong to the 19th-century zoological collection that the University of Turin has entrusted, with a special agreement, to the "Museo Regionale di Scienze Naturali" of Turin. They are currently kept for scientific purposes at the Department of Human and Animal Biology of the same university.

Unfortunately, no documented history matches the skeletons (e.g., cause of death, age at death, gender, and provenience). This information could have aided in understanding the pathogenesis of these abnormalities; in particular, data on the age at death are important in the evaluation of osseous alterations, since in thalassemia, the pattern of the bone involvement may change with advancing age. Especially in the case of isolated skeletons, some authors claim that age should be considered before any assessment of the skeletal pathologies (Moseley 1965, 1974; Lagia et al., 2007).

Considering the timing of tooth eruption in primates and according to the definition of dental development age classes, we assigned Ptr to the SUBADULT group, with an estimated chronological age ranging from 10.71 to 11.33 years and Ppy to the JUVENILE class, with an estimated age varying from 6.00 to 8.00 years (Smith, 1989; Smith et al., 1994; Swindler, 2002; Zihlman et al., 2007).

Two teeth, one 1st molar $\left(\mathrm{M}_{1}\right)$ and one 2nd molar $\left(\mathrm{M}_{2}\right)$, were pulled from the skeletons of Ptr and Ppy, respectively, and sent to the IRCCS Burlo Garofolo of Trieste for DNA extraction.

For this purpose, the edges of the dental roots of Ptr and Ppy were cut using a gear cutter; then, dental pulp was recovered using a sterile scalpel. DNA extraction was performed with the EZ1 DNA Tissue Kit (Qiagen, Valencia, CA, USA) and the EZ1 Advanced robotic workstation for automated purification of nucleic acids (Qiagen).

Genomic DNA of 10 healthy Ppy individuals from the Kuala Lumpur (Malaysia) zoo was extracted from hair roots following the protocol of Walsh et al. (1991).

The quantity and quality of genomic DNA samples were evaluated by gel electrophoresis and Nanodrop (Thermo 2000, Wilmington, DE, USA) reading.

$\beta$-globin gene mutations were analyzed by amplification using PCR and direct sequencing. Exons 1, 2, and 3 were amplified using $10 \mathrm{pM}$ of the following primers designed on the basis of the human $\beta$-globin gene sequence: first exon, forward primer: 5'-GCATAAAAGTCAGGGCAGAGC-3'; first exon, reverse primer: 5'-CCTGTCTTGTAACC TTGATACCAAC-3'; 2nd exon, forward primer: 5'-CACTGACTCTCTCTGCCTATTG3'; 2nd exon, reverse primer: 5'-CATAGAAAAGAAGGGGAAAGAAAACATC-3'; 3rd exon, forward primer: 5'-CATACCTCTTGTCTTCCTCCCA-3'; 3rd exon, reverse primer: 5'-GGAACCTTTAATAGAAATTGGACAGC-3'.

PCRs were carried out in a $25-\mu \mathrm{L}$ reaction with $0.5 \mathrm{U}$ Taq Gold (Applied Biosystems, Foster City, CA, USA), $50 \mu \mathrm{M}$ dNTPs, and $2 \mathrm{mM} \mathrm{MgCl}_{2}$. The amplification conditions were $30 \mathrm{~s}$ at $95^{\circ} \mathrm{C}, 45 \mathrm{~s}$ at $55^{\circ} \mathrm{C}$, and $30 \mathrm{~s}$ at $72^{\circ} \mathrm{C}$ for 45 cycles for exon $1,30 \mathrm{~s}$ at $95^{\circ} \mathrm{C}, 45 \mathrm{~s}$ at $55^{\circ} \mathrm{C}$, and $30 \mathrm{~s}$ at $72^{\circ} \mathrm{C}$ for 45 cycles for exon 2 , and $30 \mathrm{~s}$ at $95^{\circ} \mathrm{C}, 45 \mathrm{~s}$ at $54^{\circ} \mathrm{C}$, and $30 \mathrm{~s}$ at $72^{\circ} \mathrm{C}$ for 45 cycles for exon 3 . 

ium bromide.

PCR products were observed, under UV light, on a $2 \%$ agarose gel, stained with ethid-

DNA sequencing was performed with the Big Dye Terminator 3.1 kit (Applied Biosystems) and sequences were run on the ABI 3130 (Applied Biosystems) automatic sequencer.

$\beta$-globin sequences of Ptr, Ppy, and humans were analyzed with the "4Peaks" software (http://mekentosj.com/science/4peaks/). The CLUSTALW (Galtier et al., 1996) software allowed sequence alignment and the maximum-likelihood tree was obtained with the Mega version 5 software (http://www.megasoftware.net/).

\section{RESULTS AND DISCUSSION}

In humans, skeletal abnormalities associated with $\beta$-thalassemia include enlarged cranial and facial bones because red bone marrow over-activity determines, on the skull, the widening of the diploid space, thinning, or virtual disappearance of the outer table. Moreover, some other characteristics are: new bone formation perpendicularly to the inner table ("hairon-end" appearance), and, with regard to the inhibition of maxillary sinus pneumatization with excessive growth of the facial bones, malocclusion of the jaws, and displacement of teeth (the so-called rodent facies).

The post-cranial skeleton can show diffuse osteopenia for a generalized decrease in bone mineral density, localized areas of coarse trabeculation, widening of medullary spaces, microarchitectural deterioration, spontaneous fractures, and spinal deformities, etc. The severity of post-cranial skeletal involvement depends on the gravity of the disease and the survival of the individual to it (Moseley, 1965, 1974; Hershkovitz et al., 1997; Domrongkitchaiporn et al., 2003; Lagia et al., 2007).

In the Ptr and Ppy skeletons analyzed in our study and shown in Figure 1, some bone features were compatible with $\beta$-thalassemia. Both skeletons showed clinical signs of $\beta$-thalassemia; however, they were more pronounced in Ptr, that showed more remarkable clinical signs that were also present in Ppy.

On the skull of Ptr, extensive sieve-like porosity involved the greatest part of the components: frontal bone, the entire area of the parietals, the occipital squama, and the zygomatic processes of the temporal bones; the maxillary and mandibular bones mainly on the alveolar bone level with thinning of the cortex and trabecular coarsening. Osteoporotic spread was restricted to the outer table, while the inner surface was intact. No signs of cribra orbitalia were found.

In addition, a considerable widening of the diploid space of the calvaria bones was noted in the torus orbitalis and the lateral portion of the parietal bones with vault expansion. The sagittal and nuchal crests were remarkably developed, and at this level, radial striation of bone spicules extending through the entire width of the crests was clearly visible to the naked eye. Despite the young age at death of the individual, nearly all ecto-cranial sutures of the vault were completely obliterated, whereas all palatal, facial, and cranial-facial sutures were open, including the spheno-occipital sutures (Chopra, 1957; Winkler, 1996; Cray et al., 2008).

The facial bones were swollen, whereas the nasal bones were depressed; the upper canines were displaced forward with maxillary overbite. The mandible was less enlarged than the maxilla, likely due to its dense cortical plates and the reduced spongy bone.

On the whole, the post-cranial skeleton was damaged with generalized severe osteoporotic pitting, especially on the level of the osseous remodeling zones. The changes of the 
spine extended from the cervical area to the sacrum, with loss of bone density and accentuation and coarsening of the trabeculation of the bodies, neural arches, and spinous processes.

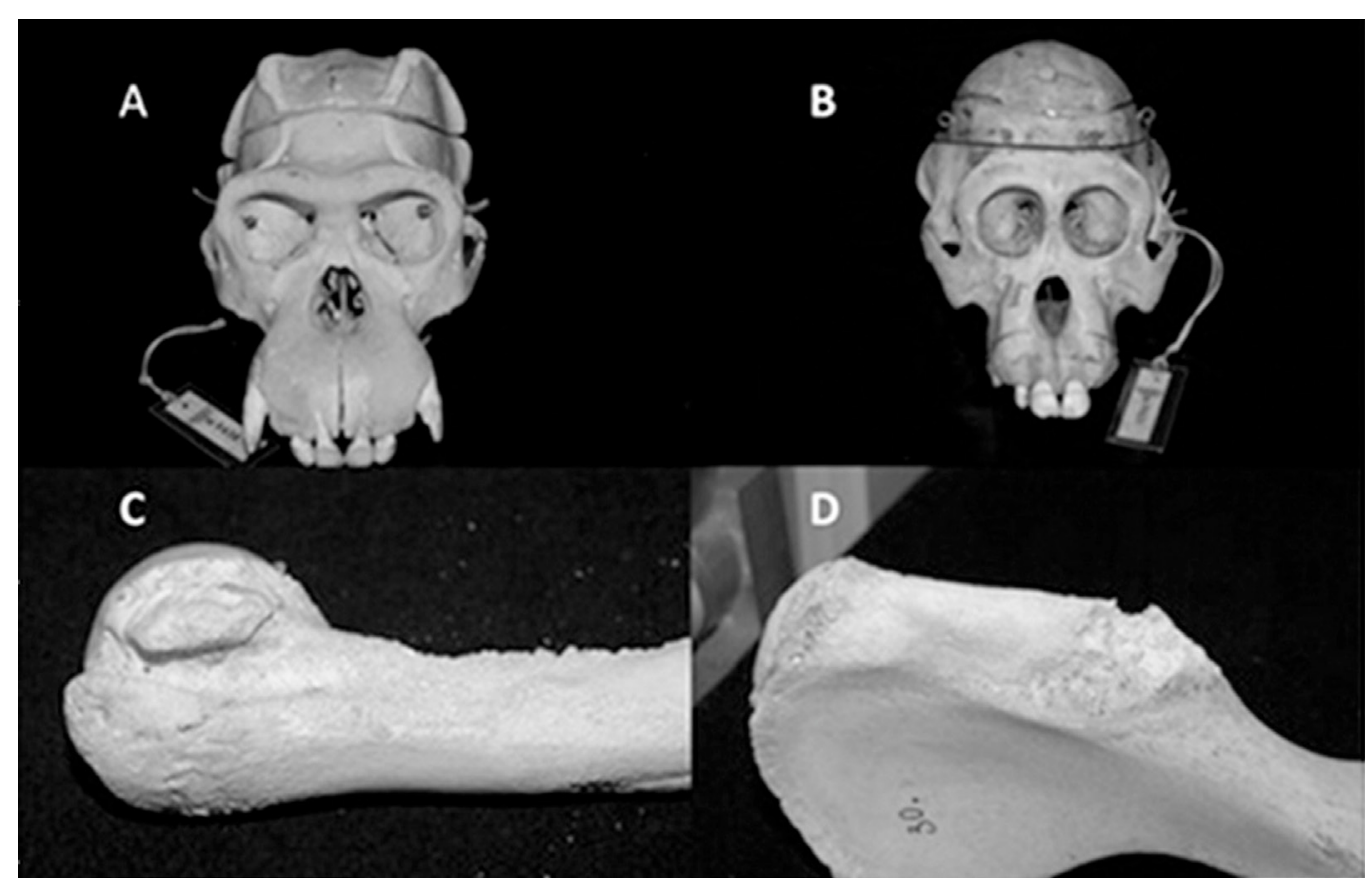

Figure 1. Morphological alterations in skulls and bones of Pan troglodytes and Pongo pygmaeus. A. Skull of P. trodlodytes; B. skull of P. pygmaeus; C. osteoporosis in homerus of P. trodlodytes; D. osteoporosis in the coxa of P. trodlodytes.

The pathological features that we recorded in the Ppy skeleton were less severe. The most serious anomalies were confined to the skull and consisted of remarkable marrow hyperplasia of the calvarial diploe, with larger overall size of the skull. Localized osteoporotic pitting of the outer compact layer of the bone involved the torus orbitalis, torus occipitalis, the alveolar bones and, to a milder degree, the temporal bones. The post-cranial skeleton showed osteoporotic signs that were mostly located at the proximal and distal ends of the long and short tubular bones.

Given the morphological clinical features suggesting the possible diagnosis of $\beta$-thalassemia, we performed a molecular analysis searching for mutations in the $\beta$-globin gene associated with the pathology of $\beta$-thalassemia.

We extracted DNA from teeth of Ptr and Ppy skeletons, conserved in a museum since 1800. We used a partially destructive technique in order to recover dental pulp from the apical root edges of the teeth, which were previously cut with a gear cutter. The lower part of the root, after being cut, was conserved and, after dental pulp recovery, the morphology of the apical root was reconstructed using dental cement.

Around $50 \mu \mathrm{L} 20 \mathrm{ng} / \mu \mathrm{L}$ genomic DNA solution was recovered from Ptr and Ppy teeth. In spite of being degraded and damaged, as expected for ancient DNA, the quality of the extracted DNA was good enough to search for mutations in the $\beta$-globin gene. We then amplified the 3 
exons of the Ptr, Ppy, and human $\beta$-globin gene. Figure 2 shows the results of the PCR amplification of exon 1 of the human, Ptr, and Ppy $\beta$-globin gene. No non-specific amplicon was detected.

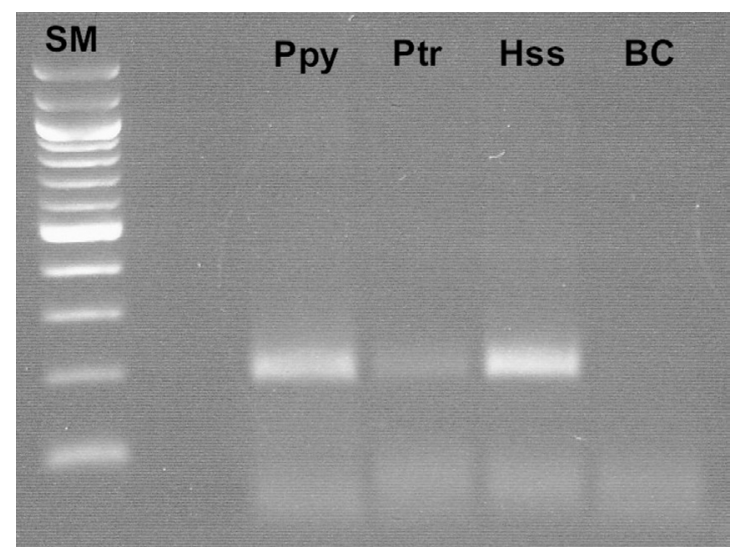

Figure 2. Gel electrophoresis of the 3 -globin gene exon 1 amplification products. Ppy = Pongo pygmaues; Hss = Homo sapiens; $\mathrm{Ptr}=$ Pan troglodytes $; \mathrm{BC}=$ blank control; $\mathrm{SM}=100$-bp molecular size marker.

Exons 1, 2, and 3 of the human, Ptr and Ppy $\beta$-globin gene were sequenced and the nucleotide sequences obtained were then aligned (Figure 3 ).

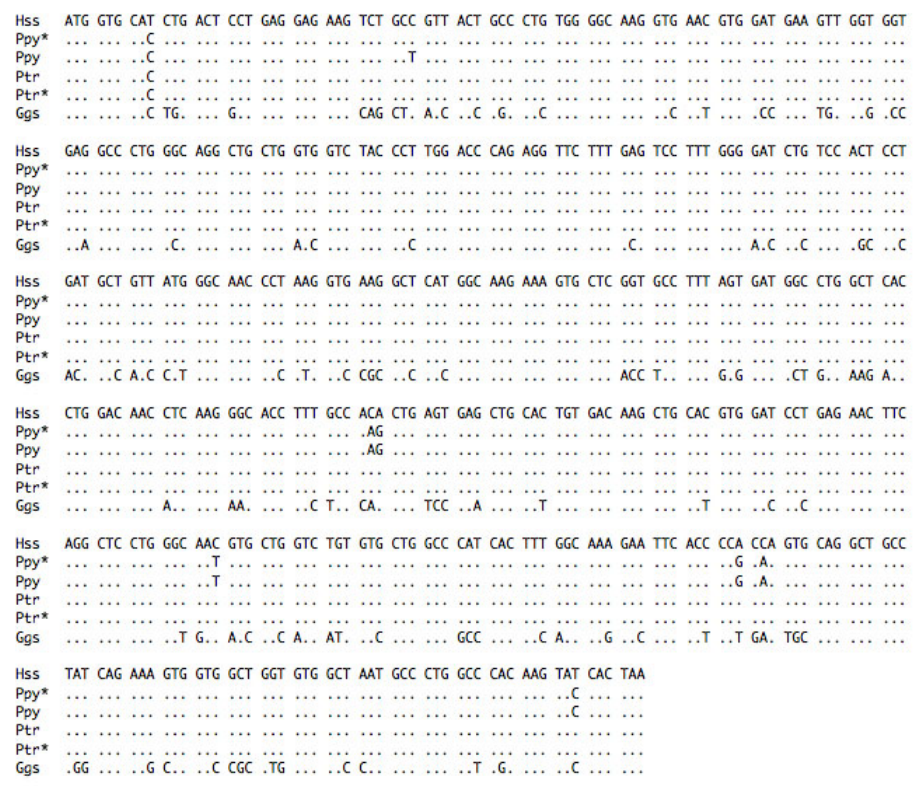

Figure 3. B-globin gene nucleotide sequence alignment of Pongo pygmaues, Pan troglodytes and Homo sapiens. Ppy $=$ P. pygmaues Ensembl reference sequence $($ ENSPPYT00000004275); Ptr $=$ P. troglodytes Ensembl reference sequence (ENSPTRT00000006177); Ppy* $=$ P. pygmaues of the present study; Ptr* $=$ P. troglodytes of the present study; Hss $=H$. sapiens Ensembl wild-type reference sequence (ENST00000335295). $\beta$-globin of Gallus gallus (Ggs) Ensembl reference sequence (ENSGALT00000028028) was used as outgroup. 
Ptr and human sequences showed the highest degree of similarity (99.8\%), while the Ppy sequence was slightly different (98.2\%). The Ppy and Ptr nucleotide sequences obtained in our study (indicated by an asterisk in Figure 3) were respectively identical to the Ppy and Ptr sequences reported in the Ensembl database, except for a $\mathrm{G}>\mathrm{T}$ substitution (silent mutation, both codons encoding for Ala) at nucleotide 33, which was present in the Ensembl Ppy sequence but not in our Ppy samples. The amino acid sequences were then deducted from the nucleotide ones and their alignment is shown in Figure 4.

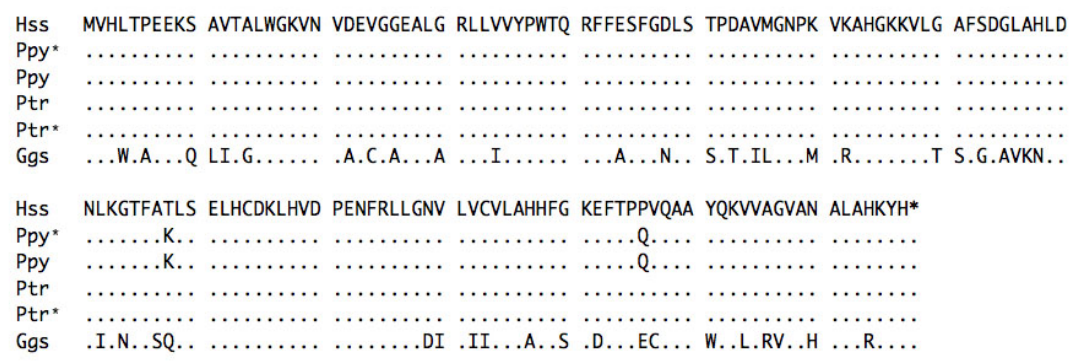

Figure 4. $\beta$-globin amino acid sequence alignment of Pongo pygmaues, Pan troglodytes and Homo sapiens. Ppy $=P$. pygmaues Ensembl reference sequence (ENSPPYP00000004109); Ptr $=$ P. troglodytes Ensembl reference sequence (ENSPTRP00000005700); Ppy* $=$. pygmaues of the present study; Ptr* $=$ P. troglodytes of the present study; Hss $=$ H. sapiens Ensembl wild-type reference sequence (ENSP00000333994). B-globin of Gallus gallus (Ggs) Ensembl reference sequence (ENSGALP00000027975) was used as outgroup.

The Ppy $\beta$-globin sequence did not present significant differences when compared to that of the 10 healthy Ppy individuals from the Kuala Lumpur zoo (data not shown).

Finally, we constructed a phylogenetic tree through the maximum-likelihood method, using the $\beta$-globin nucleotide sequence of Gallus gallus as outgroup and the human, Ptr, and Ppy $\beta$-globin gene sequences; the phylogenetic tree is shown in Figure 5.

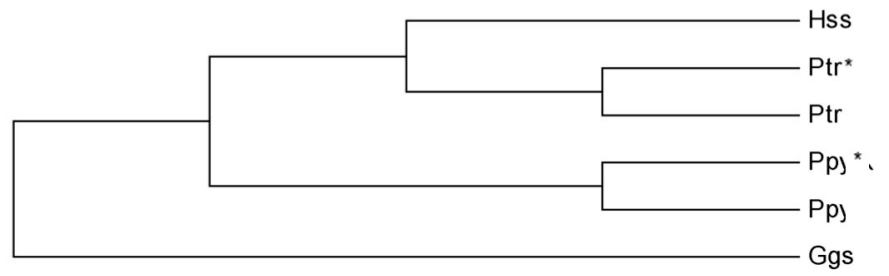

Figure 5. Maximum likelihood phylogenetic tree for great apes $\beta$-globin nucleotide sequences. Ppy $=$ Pongo pygmaues (ENSPPYT00000004275); Ptr $=$ P. troglodytes (ENSPTRT00000006177); Ppy* = P. pygmaues of the present study; Ptr* = P. troglodytes of the present study; Hss = Homo sapiens $($ ENST00000335295). Ggs $=$ Gallus gallus (ENSGALT00000028028) was used as outgroup.

The phylogenetic relationships between human, Ptr, and Ppy $\beta$-globin gene sequences were consistent with the phylogenetic tree of the great apes.

Two great apes, Ptr and Ppy, have been selected for this study since they presented 
skeletal pathologies that could be potentially associated with $\beta$-thalassemia. While Ppy only presented skull features of marked expansion of the diploic space, the entire skeleton of Ptr was affected by porotic hyperostosis, in addition to extreme bony lesions.

We successfully extracted DNA from the teeth pulp of the 2 specimens and were able to reconstruct the teeth with limited morphological damage, thereby reducing the "price" to be paid for processing museum samples. Finally, our $\beta$-globin gene molecular study has demonstrated that the 2 apes, in spite of showing some features possibly associated with $\beta$-thalassemia, did not present the molecular defects typically associated with the disease in humans.

\title{
ACKNOWLEDGMENTS
}

\author{
We wish to thank Prof. G. Ardito, Dr. A. Mottura, and Dr. R. Boano for their valuable \\ suggestions.
}

\section{REFERENCES}

Chopra SRK (1957). The cranial suture closure in monkeys. Proc. Zoolog. Soc. London 128: 67-112.

Cray J Jr, Meindl RS, Sherwood CC and Lovejoy CO (2008). Ectocranial suture closure in Pan troglodytes and Gorilla gorilla: pattern and phylogeny. Am. J. Phys. Anthropol. 136: 394-399.

De Mattia D, Pettini PL, Sabato V, Rubini G, et al. (1996). Oromaxillofacial changes in thalassemia major. Minerva Pediatr. 48: 11-20.

DeGusta D (2010). Cribra Orbitalia: A non-human primate perspective. Int. J. Osteoarchaeol. 20: 597-602.

Domrongkitchaiporn S, Sirikulchayanonta V, Angchaisuksiri P, Stitchantrakul W, et al. (2003). Abnormalities in bone mineral density and bone histology in thalassemia. J. Bone Miner. Res. 18: 1682-1688.

Galtier N, Gouy M and Gautier C (1996). SEAVIEW and PHYLO_WIN: two graphic tools for sequence alignment and molecular phylogeny. Comput. Appl. Biosci. 12: 543-548.

Hershkovitz I, Rothschild BM, Latimer B, Dutour O, et al. (1997). Recognition of sickle cell anemia in skeletal remains of children. Am. J. Phys. Anthropol. 104: 213-226.

Hes J, van der Wall I and de Man K (1990). Bimaxillary hyperplasia: the facial expression of homozygous beta-thalassemia. Oral Surg. Oral Med. Oral Pathol. 69: 185-190.

Hollar MA (2001). The hair-on-end sign. Radiology 221: 347-348.

Lagia A, Eliopoulos C and Manolis S (2007). Thalassemia: Macroscopic and Radiological Study of a Case. Int. J. Osteoarchaeol.17: 269-285.

Miller W, Drautz DI, Ratan A, Pusey B, et al. (2008). Sequencing the nuclear genome of the extinct woolly mammoth. Nature 456: 387-390.

Moseley JE (1965). The paleopathologic riddle of "symmetrical osteoporosis". Am. J. Roentgenol. Radium Ther. Nucl. Med. 95: 135-142.

Moseley JE (1974). Skeletal changes in the anemias. Semin. Roentgenol. 9: 169-184.

Nathan H and Haas N (1966). On the presence of cribra orbitalia in apes and monkeys. Am. J. Phys. Anthropol. 24: 351-359.

Rascol VL, Pontarotti P and Levasseur A (2007). Ancestral animal genomes reconstruction. Curr. Opin. Immunol. 19: 542-546.

Rohland N, Siedel H and Hofreiter M (2004). Nondestructive DNA extraction method for mitochondrial DNA analyses of museum specimens. Biotechniques 36: 814-821.

Rohland N and Hofreiter M (2007a). Ancient DNA extraction from bones and teeth. Nat. Protoc. 2: 1756-1762.

Rohland N and Hofreiter M (2007b). Comparison and optimization of ancient DNA extraction. Biotechniques 42: 343-352.

Roy MS, Girman DJ, Taylor AC and Wayne RK (1994). The use of museum specimens to reconstruct the genetic variability and relationships of extinct populations. Experientia 50: 551-557.

Schultz AH (1956). The occurrence and frequency of pathological and teratological conditions and of twinning among non-human Primates. Primatologia 1: 965-1014.

Smith BH (1989). Dental development as a measure of life history in primates. Evolution 43: 683-688.

Smith BH, Crummett TL and Brandt KL (1994). Ages of eruption of primate teeth: a compendium for aging individuals and comparing life histories. Am. J. Physical Anthropol. 37: 177-231. 
Stuart-Macadam P (1987). A radiographic study of porotic hyperostosis. Am. J. Phys. Anthropol. 74: 511-520.

Swindler DR (2002). Primate Dentition: An Introduction to the Teeth of Non-Human Primates. Cambridge University Press, Cambridge.

Walsh PS, Metzger DA and Higuchi R (1991). Chelex 100 as a medium for simple extraction of DNA for PCR-based typing from forensic material. Biotechniques 10: 506-513.

Willerslev E and Cooper A (2005). Ancient DNA. Proc. Biol. Sci. 272: 3-16.

Winkler LA (1996). Appearance of ossification centers of the lower arm, wrist, lower leg, and ankle in immature orangutans and chimpanzees with an assessment of the relationship of ossification to dental development. Am. $J$. Phys. Anthropol. 99: 191-203.

Zihlman AL, Bolter DR and Boesch C (2007). Skeletal and dental growth and development in chimpanzees of the Taï National Park, Côte D’Ivoire. J. Zool. 273: 63-73. 